\title{
Mental Health Insights from Individual Physiologic and Lifestyle Continuous Sensing
}

\author{
Tabya Sultan* \\ Maximillian Lin \\ University of California, Irvine
}

\author{
Nitish Nag* \\ Zepp USA Research
}

\author{
Alex Richardson \\ University of California, Irvine
}

\begin{abstract}
Mental wellness provides an essential foundation for healthy living. Traditionally, mental health is primarily measured subjectively and understood as a categorical disease based on certain criteria. Recent shifts in psychological sciences call for assessing mental health more objectively along a quantifiable spectrum. This paradigm allows for closer to real-time understanding of mental status. Modern ubiquitous sensing technologies that capture objective data streams provide an opportunity to track mental health related parameters continuously and quantitatively. This allows understanding mental health more deeply through encompassing holistic lifestyle aspects that have been previously challenging to measure due to Hawthorn effect, user burden, and subjective assessment limitations. This work focuses on two concepts. First, we emphasize the various semantic concepts of mental health on fluid quantitative continuum and how this can be translated to a computing framework. Second, we utilize non-invasive continuous personal quantitative data streams to assess markers of mental health state along various parameters. We define the mental health state insight framework to 1) compute parameters related to the current mental health state and 2) determine if a user's life events and physiologic factors are helping or harming their mental health state. We conduct a pilot study with retrospective in-situ data tracking to illustrate the utility of our analysis framework. We conclude by proposing how this information can inform users and healthcare providers to make better-informed decisions to improve the mental health care journey.
\end{abstract}

\section{KEYWORDS}

health state estimation, data-driven mental health, wearables, ubiquitous sensing, personalized health, precision health, preventive health, multi-modal health

\section{INTRODUCTION AND RELATED WORK}

Mental health has been defined by the World Health Organization as "a state of well-being in which the individual realizes his or her own abilities, can cope with the normal stresses of life, can work productively and fruitfully, and is able to make a contribution to

Permission to make digital or hard copies of all or part of this work for personal or classroom use is granted without fee provided that copies are not made or distributed for profit or commercial advantage and that copies bear this notice and the full citation on the first page. Abstracting with credit is permitted.

${ }^{*}$ Authors contributed equally to this work.

1 ,

(C) 2021

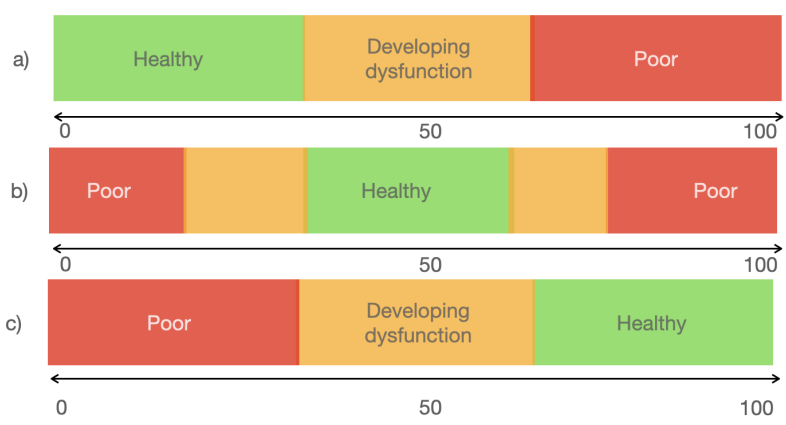

Figure 1: Dimension model types: In spectrum a) Lowest level of parameter is healthiest. Example: Substance use in which too much substance may only harm one's health. Spectrum (b), reflects an optimal healthy balance point for this parameter. Example: Energy levels, where too much (like in mania) or too little (like in depression) is unhealthy. Spectrum (c) shows that more of a certain parameter is healthier. Example: Too little satisfaction of life may lead to depression or isolation, but increasing amounts are beneficial to the individual. Observing parameters that can estimate the rate of change along these dimensions is the primary goal of this work.

his or her community" [29]. This definition has since expanded to include the dynamic nature of an individual's internal equilibrium. This includes one's ability to socialize, recognize, express emotions, empathize, provide flexibility to cope, and provide a harmonious relationship between the body and mind [29].

Diagnosed mental disorders have recently shifted towards being defined as a position within a spectrum, rather than specific to a categorical disease state $[3,53]$. Within psychiatry, conditions are less often categorized as either having the condition or not and more so described along a spectrum with modifiers of severity [3]. Most psychologists and mental health professionals acknowledge, understand, and measure the mental health continuum of higher or lower symptom severity. This continuum is documented in the National Institute of Mental Health's Research Domain Criteria (RDoC), integrating many levels of information to explore basic dimensions of functioning that span across the full range of human behavior [58].

A historical challenge within the field of mental health research, is the lack of granular and objective data about the mental health status of an individual. Such data would allow for better quantification of the mental health state, improvement on guidance for 
an individual and tracking effective changes, other than through surveys and subjective evaluation methods. There can frequently be a divergence in what a person reports and what is occurring (e.g., in a manic episode). As the mental state is dynamic, measurements to determine the state must also be appropriately frequent to capture these changes. Alluding to Nyquust rate theorum, to effectively capture the dynamic change of a function $x(t)$ without aliasing effects, data sampling rate must be captured at a minimum frequency (f) of greater than twice the rate of the highest bandwidth $(b)$ of change:

$$
f>2 b
$$

Given mental status changes of various types (energy, focus, stress, mood, sleep patterns, etc.) change at various rates, sampling would need to be done at least more than twice as frequent as changes expected in the mental status variable of choice. Two direct measurement categories include the individual's biological mental status and lifestyle events. Combined these can both be used as indicators of personal mental health status. Fortunately, with the advent of sensor technologies, we can begin to capture signals that can satisfy the Nyquist rate. Furthermore, by measuring the rate of change regarding the various signals, we can gain greater insight into the future trajectory of the signal.

The increasingly ubiquitous use of wearables and smart devices has made ecological momentary assessment (EMA) an increasingly feasible method for rich subjective data collection in mental health $[13,64,71]$. EMA has increasingly been utilized to study emotional and mental states for clinical assessment of psychopathology in outpatient settings due to the superior temporal resolution and accuracy compared to traditional, retrospective survey-based methods. This can be combined with measures that sample a subjects' behavioral and physiological data in real-time and real-world settings.

The development of new sensor technologies has brought about the growth in personal sensing research within mental health. While still a growing area in research, we see the use of ubiquitous and continuous sensing methods, such as smartphones, social media, and computers, that are used in translating raw sensor data into markers of behaviors and states related to mental health [52] As an example, computational machine learning techniques have been applied to screen for depression using images uploaded to an individual's Instagram account with diagnostic accuracy outperforming general practitioners' average unassisted diagnostic success rate [67]. These biomarkers are fundamental in predicting sleep, mood, and stress and serve as indicators for clinical disorders such as depression, schizophrenia, and bipolar disorder [52]. Utilizing sensor technologies allow us to produce large amounts of data and aggregate all unique data fields on one's life to detect sub-optimal mental states. Sensors may contribute to empowering providers to help individuals navigate personal goals, as exemplified by recommender systems that take a user's model to make recommendations towards improvement $[55,66]$.

There have been efforts on understanding personal events, for example, life-logging focused on mental health [37, 38, 59]. However, techniques or tools that evaluate deep episodic data and produce correct diagnoses with appropriate action plans for mental health are lacking.

Furthermore, the concept of data-driven personalization in psychiatry is growing. The information we receive through technology allows for diagnostic clarification, symptom tracking, and reinforcing beneficial behaviors of those with mental illness [53]. Computing on this data can facilitate guidance towards better mental health through modern personal health navigation computing methods [55].

Current frameworks for sensor-based data collection in mental health include digital phenotyping, which collects data from smart devices to build a rich personalized picture of behavior, track markers of mental health conditions, choose treatments, and more $[10,35,36,74,77]$. Digital phenotyping has traditionally been described as behavior pattern mining through sensors. Technically speaking, phenotypes refer to the gene expression profile of a biological entity. Phenotypes are generally more stable over the course of time, such as in personality and behaviors. This traditional digital phenotyping can be used as a tool to uncover the input-output response model in more depth about a specific individual, allowing for personalized mental health. In contrast, mental health state is a much more dynamic, rapidly fluctuating response to inputs.

Within this research, we aim to present a conceptual and pilot demonstration that combines key parameters related to mental health with the continuous sensing measurements. By observing the first derivative of the moving averages regarding these parameters, we hope to better understand an individual's dynamic mental state. The user and provider can then employ this information to investigate further changes in behavior, therapy needs, and the change in mental health of an individual. Additionally, this information could be utilized in a preventive capacity to monitor mental health in individuals experiencing mental distress or symptomatology that does not quite meet diagnostic criteria for a mental health disorder. Our research aims to use measurements of mental health biomarkers in personalized, data-driven approaches to evaluating the various dimensions of mental health for each unique user.

\section{THE MENTAL HEALTH STATE SPECTRUM}

Knowing a user's mental health state provides information about one's health situation. The events in one's life become experiences, which are valuable for determining where an individual is on the spectrum. Mental health is a dynamic state continually changing based on biology, the environment, life events, and unique behavioral patterns. Technology that senses a change in a user's position on the mental health spectrum is essential to improve one's health [56]. In order to measure where a user is within the state space there are three key steps: 1) define the chosen relevant dimensions of mental health, 2) apply the dimensions specific to the user, and 3) estimate precisely where an individual is located within the state space defined by the aforementioned dimensions.

2.0.1 Dimensions in Mental Health. First, we must define clearly the dimensions in which we will be measuring the mental state. These dimensions are along a spectrum, allowing us to understand the severity, and rate of change (first derivative) and acceleration (second derivative) of the individual within that dimension. For example, if we look at a user's ability to focus, there are two ends 
of the spectrum: on one end, a user is unable to focus at all, as related to attention deficit disorder (ADHD), and the other end of the spectrum is when a user has too much focus, as related to obsessive-compulsive personality disorder (OCPD). In the middle of this spectrum would be "healthy attention." The concept of this spectrum approach allows us to quantify where an individual would reside on this spectrum and be able to indicate a healthy state, developing dysfunction, or impairment in day-to-day functioning. The regions within a spectrum may be classified depending on the condition or mental status. Each dimension can be labeled with semantic thresholds in which users would be in a state of "optimal," "disease," or "prodromal." We will be discussing various examples throughout our work, but the general principle of the spectrum approach is described in Figure 1.

There are several dimensions to consider [3] and may be clinically oriented around conditions, such as depression, anxiety, psychosis, and focus. All the relevant dimensions added together creates a personal state space for a unique user and context.

\subsection{Mental Health State Space}

We can now define these dimensions within one high-dimensional space, which we call the mental health state space. In theory, this space would contain all relevant dimensions that may interact with mental health. Each person has a unique and finite possibility of states given their biology, life events, cognitive functioning, mood, and behavior. These finite regions are established by placing relevant upper and lower bounds on each dimension giving rise to a personal mental health state space for the user's situation.

\subsection{Mental Health State Estimation}

Next, we define the user's position within the state space. It is critical to determine the aggregation of relevant dimensions to represent the state and determine where this is relative to a user's baseline. We can think of the location as GPS coordinates of the space, which gives us a starting point in their health journey. It is important to note that the user may be in constant flux given life changes. Thus, the mental health state estimation gives a summary of a user's mental health status through quantitative assessments by fusing data and domain knowledge within the dimensions of mental health in a given time frame $[46,56]$. Estimation techniques have been essential for mental health applications, but are more effective combined with biological knowledge and family history (e.g., age, genetics) of their mental state. Techniques combining low and highquality data streams can improve the accuracy of the mental health state estimated location through methods such as Kalman filtering, GPS/INS integration [23, 65] and reinforcement learning [4]. We can also apply individual characteristics (i.e., genetics, sex, age) to provide boundaries to the thresholds of the state space.

\section{MENTAL HEALTH INSIGHT SYSTEM FRAMEWORK}

In order to understand where a user is within their mental health state space, we must build a system for tracking lifestyle dimensions and changes over time. Within our framework, we aim to understand where a user is relative to their baseline, see how far a user is from their goals, and determine if activities or events in the user's life are changing their state.

\subsection{Measurements for Tracking}

In order to measure a user's position in the mental health state space, we need to collect various types of data about the user. We use a combination of hardware and software, alongside public data resources and open source-based tools, to collect data about the user within the scope of mental health. Measurements are made on an automatic continuous basis or by volitional user inputs. Continuous sensors eliminate the barrier to actively engaging in measurements as the data is collected constantly, reducing user burden. Once inputs are needed, the measurements become more cumbersome for the user. However, both are essential to generate high-resolution user data.

Continuous sensors, digital interactions, and biological measurement devices produce vast amounts of data on an individual's perception, biology, environment, and life events. Continuous digital sensors, including computers, smartphones, and wearables, are relatively inexpensive in cost, energy, and computing power. Data quality and collection frequency should be appropriately monitored. The advancement of these technologies makes them widely available for health monitoring [47].

We investigate various measurement tools related to lifestyle events as part of a user's day-to-day functioning. The hardware and software data sources will capture low-level behavioral health factors such as sleep, screen usage, physical activity, location, and environment, as described in 2 . The data sources will serve as biomarkers that will be measured via events to better understand a user's lower-level dimensions, including energy, stress, mood, sleep quality, and social environment. It is important to note that a consortium of parameters can be uniquely combined for each psychiatric mental health state. For example, analyzing patterns in sleep is vital for both mania and depression.

\subsection{Event Extraction and Analysis}

We will now review measurable life events a user partakes related to changes in their mental state.

3.2.1 Sleep. Sleep pattern alterations are a common symptom in several mental health and psychiatric conditions [28]. More importantly, insomnia has been found to be associated with, or predictive of multiple mental health disorders, including anxiety and depression $[11,63,68]$ with several studies demonstrating a causal relationship between insomnia and depression or anxiety [28, 39]. We can see disturbances based on how long a patient is sleeping and their sleep quality. Sensors in an iPhone can determine sleep duration through accelerometer, microphone, ambient light sensors, battery state, screen proximity sensor, and display screen state [20]. Sleep period markers can contribute to circadian awareness (i.e., sleep time of day, sleep pattern by day). We can also determine factors that impact sleep, including noise and snoring, using a microphone in a smartphone. We also can combine data from an individual with domain knowledge relevant for the user to set goals for improving sleep. For example, sleep disorders, such as narcolepsy and obstructive sleep apnea, may be more prevalent in individuals with genetic predispositions [70]. 


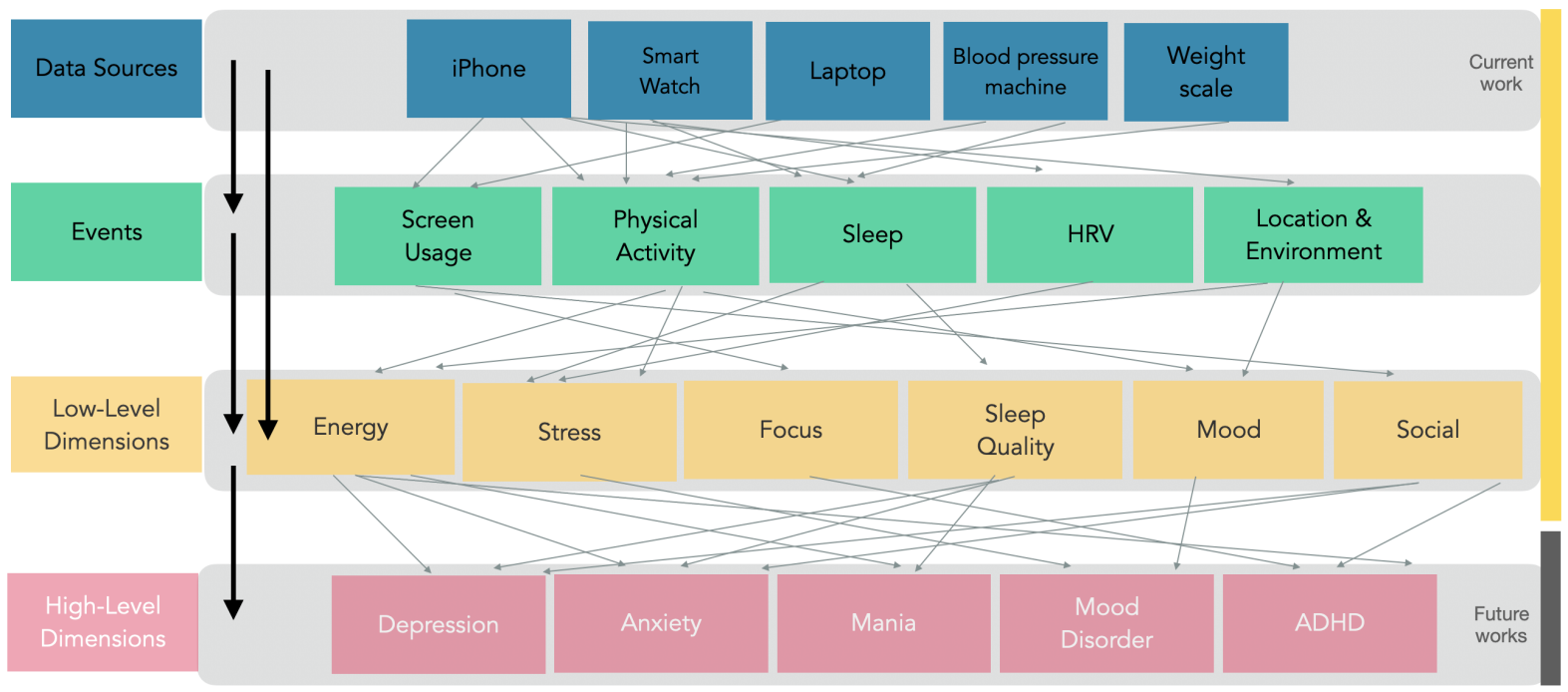

Figure 2: Framework: The mental health estimation framework has multiple layers; 1 . Data sources from various sensors, 2. Events that can be tracked given the various sensors, 3. The low-level dimensions in which the individual is experiencing a mental health situation, 4 . The high-level dimensions in which an individual is diagnosed or classified based on symptoms and states. We focus on layers 1 through 3 within this work.

3.2.2 Screen Usage. Screen usage habits indicate one's ability to focus more productive hours on their screen. Studies conducted show that decreased screen time and increased physical activity are associated with positive effects to mental health [16, 25, 45]. Productivity measuring systems, like RescueTime, track hours on screen and can indicate if the type of online content is contributing to one's productivity or if it is spent on distractions or entertainment [5]. If we take the dimension of Focus, which is critical for conditions including OCPD or autism spectrum disorder, we look at ways to measure an individual's ability to focus and see if they may be improving, getting more distracted, or stable. For example, RescueTime can track all websites and time spent on one's phone and desktop. We can also aggregate data on phone pick-ups and the duration of interactions with one's iPhone. Seeing how the user behaves allows providers to gain insight into patient activities beyond the user sharing knowledge or personal experiences that may not be captured accurately. Other trackers include ScreenTime on one's smartphone, which captures the number of times one picks up their phone and for what purpose.

3.2.3 Physical Activity. Physical activity is an important component in the management of mental health conditions such as depression and anxiety. Physical inactivity has been described as a risk factor for developing depression, or anxiety [15, 73, 76], and it has been linked with higher self-reported mental health burden [19]. Increased aerobic exercise or strength training has been shown to reduce symptoms of depression and anxiety significantly $[27,40,44,60]$, as well as to increase resilience to acute stress [21]. There is a suggestion that the beneficial effects of exercise may vary based on intensity. Moderate continuous training decreases depressive symptoms and serum levels of the proinflammatory cytokine tumor necrosis factor alpha (TNF-alpha), while high-intensity interval training decreases depressive symptoms but simultaneously increases perceived stress along with serum TNF-alpha and interleukin-6 (IL-6), another pro-inflammatory cytokine [61]. Similarly, moderate-intensity anaerobic training was superior to both low and high-intensity anaerobic training for mood improvement, though data on aerobic activity intensity was less clear [18]. Any amount of physical activity can be beneficial for mental health by protecting against depression [22]. Therefore, tracking of general activity and the intensity of exercise can be an indicator of mood, disease state, and any changes an individual is making to impact their mental health. There are several trackers of physical activity that are widely available, including activity trackers within the smartphones and steps recorded in wearables [7]. The intensity of exercise can be captured by heart rate measurements in wearables and heart rate monitors. We can also measure longer-term physiological factors, such as blood pressure, which may indicate stress over time. Blood pressure is susceptible to change depending on activity levels.

\subsection{Biological State Extraction and Analysis}

3.3.1 Heart Rate Variability. Heart rate variability (HRV) is a psychophysiological marker for mental health and physical well-being and is widely considered to be a measure of emotional regulatory capacity ([78]). Fluctuations in HRV, or the time between successive heartbeats, are thought to reflect the competing influences of the sympathetic and parasympathetic divisions of the autonomic nervous system, with reductions in HRV indicating decreased parasympathetic vagal tone. For example, reduced HRV has important functional significance for motivation to engage in social situations, psychological flexibility when faced with a stressor, and in patients with mood disorders [41]. Reduced heart rate variability 
has been reported in a variety of psychiatric disorders including major depressive disorder [41], anxiety disorders [17], schizophrenia [24], bipolar disorder [54], borderline personality disorder [42], attention-deficit hyperactivity disorder [14] and conduct disorder [12]. HRV can be an important indicator of how stressed an individual is, and it can also be an indicator of the development of psychiatric conditions based on trends over time. However, it is important to note that there may be confounding factors in other physical health realms that contribute to changes in HRV. While low HRV may be a marker of a diverse array of psychopathologies, some psychopathologies such as anorexia nervosa have been associated with high HRV [49]. Additionally, high HRV has been reported in physically over-trained endurance athletes [80], and abnormally elevated HRV in the elderly population has been associated with a higher risk of mortality [75]. It has been suggested that an ideal range of HRV exists for optimal performance [32], but it may be most insightful to view relative trends in HRV and interpret changes contextually while using large-scale cross-sectional studies of HRV data to help define the reference range [57].

3.3.2 Location and Environment. A user's GPS location data through a phone or wearable allows us to determine where the individual is located at a given time. This provides us knowledge of circadian patterns based on sunrise and sunset by location. In locations with limited sunlight during the day, individuals are more likely to develop seasonal affective disorder (SAD). Circadian rhythms are also an important factor in light-dark cycles, contributing to one's mood [79]. Devices are controlling ambient lighting, such as Phillips Hue light bulbs, or temperature-controlled sensors, such as Nest [6, 31]. Movement based on geolocation data is also linked to mood and depression [33]. Greater daily variability in location is associated with increased positive affect. Location is also able to provide data regarding environmental exposures, such as pollutants. Long-term exposure to ambient air pollution may be an independent risk factor for mental health disorders ranging from subjective stress to suicidal ideation [72]. Based on GPS location, we can know more generally which pollutants an individual is exposed to on an ongoing basis.

\subsection{Integrated High-Level Mental State}

For each condition, there may be certain high-level features relevant for the user. For example, with depression, we know that sleep, energy, and stress are indicators of disease severity [43]. We can build a system to see where an individual is located relative to the baseline for each area by using measurements related to sleep, physical activity, HRV, and more. This work does not extend into the scope of creating a total integrated view, but is a logical next step for future works.

\section{EXPERIMENTAL METHODS}

We apply the mental health state parameter framework into a specific application to test the utility in real world users. We test how various measurements can generate a meaningful analysis of a user's mental state, especially through the analysis of the first derivative of each parameter. Primarily, we look at if the first derivative is positive, zero, or negative, reflecting a sustained trend in the parameter. We also demarcate verified outliers from these trends and investigate with the user the causal mechanism of such a deviation.

\subsection{Feasibility}

First, we must look at the feasibility of the mental health estimation framework in a real-world setting to ensure that the concept can be applied to widespread users. We want to understand the hardware, the cost to the user, convenience of use, data standardization of the various data captured, the feasibility to incorporate with established clinical platforms and systems, and the accuracy of the data itself.

4.1.1 Hardware. Ultimately, at a start, just having a smartphone and a wearable device can provide multiple data points on a user's life events 1 including sleep, physical activity, stress, and productivity. Already, 85 percent of the U.S. population uses a smartphone, and 21 percent of the U.S. population owns a smartwatch [1,9]. This makes the hardware widely available to a large segment of the population.

4.1.2 Cost. The cost of a psychiatrist visit in the United States is approximately between 100-300 dollars per visit [50]. Much of the consultation includes psychiatrists inquiring about changes in a user's lifestyle and habits in the last couple of weeks since the prior visit. With the mental health estimation system, providers' time can be greatly reduced by generating automatic patient trends. The cost of a wearable device to capture all the data components proposed within our research is approximately a one-time fee of 50-200 dollars, including devices like Garmin, FitBit, Apple Watch, and more. Furthermore, the perceived cost of wearables has no significant effects on user intention to use the devices [62].

4.1.3 Convenience. A potential barrier to data-driven mental health care is users' inputs into tracking. The more seamless and automatic the data collection process can be, the more likely users will agree to track their mental health, and the higher their compliance will be. Eliminating inputs from the user also reduces reminders in which an individual has to take a particular action. This will produce more consistent data over time, given the continuous nature of the devices used. Other devices provide higher resolution data, such as a CorSense HRV device; however, it is an additional device and requires a user to take a reading to determine the HRV, rather than automatically from a smartwatch. Here, providers would need to weigh the importance of user convenience versus data quality.

4.1.4 Data standardization. The communication and data streams should not be propriety to be most widely circulated and useful for providers globally. Both Apple and Google are contributing to greater data standardization. Apple built a Health Records section into its own native Health app in iOS [2]. The standard data points on an individuals' health (e.g., weight, blood pressure) can now be universally captured regardless of the wearable device a user purchases. Data standardization allows for fewer barriers to access. It is also important to integrate with existing clinical platforms to remove friction for providers, such as Fast Healthcare Interoperability Resources (FHIR). FHIR describes a set of standards that facilitate the transfer of healthcare information between electronic healthcare systems and is becoming a standard in psychiatry today [8]. This system allows for more collaborative models of care, and 
all individual data can be incorporated into this existing electronic health system.

4.1.5 Data Accuracy. Next, we look at how accurate the data is that is being produced. There may be errors oriented around the consistency of use. For example, users not consistently wearing a watch at night during sleep can give readings of null values. This then indicates a zero-hour sleep night which will skew the data and averages and give falsely low averages. As data processing techniques advance, we anticipate there will be fewer challenges with data accuracy. However, current evidence shows that consumer wearable devices, notably the Apple Watch, have a high degree of accuracy when deriving parameters such as sleep, activity levels, and HRV-based parasympathetic tone [30, 34, 51, 69].

\subsection{Case Studies}

4.2.1 Experimental Setup. We analyzed four unique users using lifestyle data to see any correlations between the data and the user's mental health. We collected user life event data through an iPhone and smartwatch (Garmin or Apple Watch) within 5 to 9 months. Our objective was to determine whether or not there are meaningful trends presented in the lifestyle data that a provider can use to gain information on a patient's condition progress and behavioral patterns.

We collected user data given various sensors to determine patterns about the user 1. We used a combination of smartphone sleep tracking (e.g., Sleep Cycle) and a smartwatch for sleep events. We were then able to see trends on time in bed, time asleep, the number of awakenings, and wake-up time. These metrics give insight into user sleep quality and consistency. For physical activity, we used the iPhone to measure steps and stairs throughout the day and the smartwatch to track heart rate and activity intensity. We used both the smartwatch and an HRV tracking device, CorSense HRV, to measure changes in HRV. We used SDNN as the unit for measuring $\mathrm{HRV}$ as this is also the main unit for HRV in Apple Health Kit. It is important to note that while HRV has a relationship with stress, it is also affected by other factors including caffeine, alcohol, etc. These confounding factors are not taken into account in our experimental setup. Next, we used an iPhone to track screen time and the number of phone pick-ups per day to analyze productivity. We used the software, RescueTime, to measure total time on screen and productivity metrics related to screen activity.

Table 1 are the various data components we looked at over a shorter time frame (7-day average) and longer time frame (42-day moving average). The measurements per week in the 7-day average will help understand the variability of sleep and physical activity in the short term compared to the user's longer-term baseline. The data can also help determine whether an individual is improving and what may be the variables contributing to the improvement. The 42-day moving average can also indicate sustained trends over time rather than sporadic changes.

4.2.2 Data Trends. After capturing all of the data for a given time frame, we aggregated the results and visualized them within an activity data analysis platform, Intervals.icu. User data included HRV, blood pressure and weight, exercise load, cardiorespiratory fitness (CRF), and sleep. Because devices were unique to each user,
Table 1: Data Source Measurements

\begin{tabular}{lll}
\hline Life Event & Data & Tool \\
\hline Sleep & Time in Bed & iPhone Sleep Tracker, Smart Watch \\
& Time Asleep & iPhone Sleep Tracker, Smart Watch \\
& \# of Awakenings & iPhone Sleep Tracker, Smart Watch \\
& Wake up time & iPhone Sleep Tracker, Smart Watch \\
Activity & Steps & iPhone \\
& Stairs & iPhone \\
& Heart Rate & Smart Watch \\
& Weight & Weight scale \\
Stress & HRV & Smart Watch, CorSense HRV \\
& Blood Pressure & Blood pressure cuff
\end{tabular}

Table 2: Data sources used in the evaluation parameters that can compose events and low-level dimensions of the experimental case studies.

not all measurements were captured for all users. We also captured any notable life events users experienced during their data collection period. These events were estimated retrospectively by the user in an interview process with questions inquiring what events occurred during the period of time with notable changes in the lifestyle data. Life events identified by users provided knowledge on life circumstances that may impact the data.

We reviewed each user dataset with the following steps that allow us to analyze key insights that would be relevant to a user and their mental health provider:

(1) Check data quality.

(2) Observe 42-day moving average long term trends.

(3) Identify inflection points in long term trends.

(4) Check if inflection points correlate with life event.

(5) Check if inflection points relate to improvement or reduction in health state.

(6) Observe 7-day moving average short term trends.

(7) Identify inflection points and variability change points.

(8) Inquire with user on causality for inflection point or variability change point through interview process.

(9) Inquire with user if short term change points relate to improvement or reduction in health state.

4.2.3 User 1: Case Study. The results for User 1 is shown in 3.

For User 1, the HRV results show the stress an individual may be experiencing over the last six months. From March to April, we see a significant, sustained increase in HRV (Fig. 3a), and the trend continues to be a long-term improvement (Fig. 3b) as shown in the 42-day moving average. We then see that starting June, the average slowly declines (Fig. 3c). Within the six months, we observed critical life events, including the start of school in June. The analysis indicates that there may be stressful circumstances in the new work environment. Lastly, the sudden tapering of HRV continues to decline when long work hours begin in late July. We note there is rebound parasympathetic in HRV after a period (Fig. 3d) of sleep deprivation (Fig. 3p). It is important to note that HRV may have 


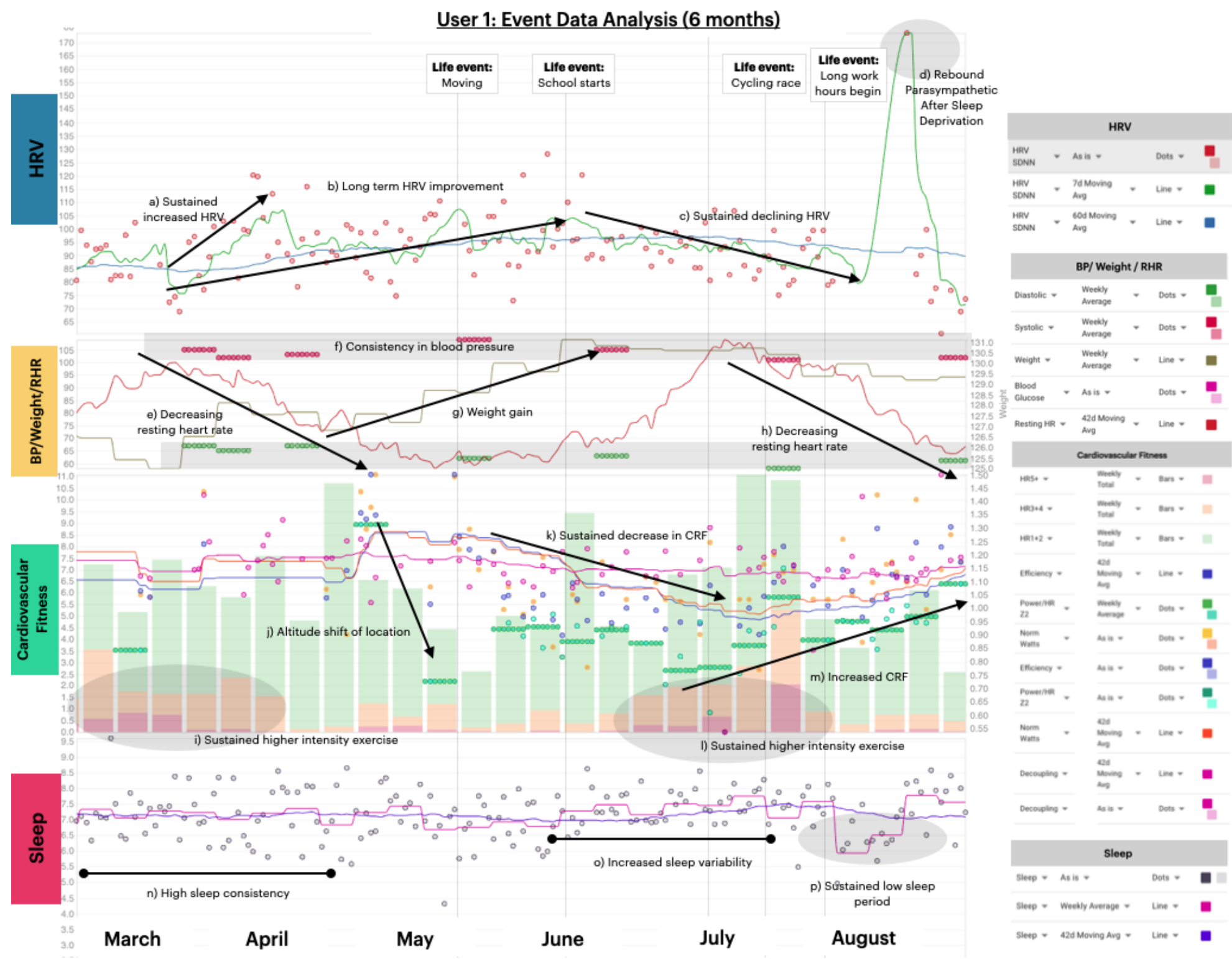

Figure 3: User 1 Data Trends: We analyzed 6 months of data for User 1, capturing trends in HRV, blood pressure, weight, resting heart rate, $\mathrm{CRF}$, and sleep. Labels a through $\mathrm{k}$ provide relevant trends and insights from both 7-day moving averages and 42-day moving averages.

confounding factors with CRF. For example, HRV may decrease when there is high-intensity exercise (Fig. $3 \mathrm{~m}$ ).

Next, we observed changes in User 1's blood pressure and weight over six months. From April to June, we see constant weight gain (Fig. 3g). This change may indicate behavioral changes or changes to diet, important for conditions like Depression. Diastolic and systolic blood pressure constantly remain low over time 105/60 mm Hg (Fig 3f). It is important to note that changes in blood pressure can be an indicator of high stress over time [48]. Lastly, we observed a decreasing resting heart rate from March to May and July to August, an indicator of increased respiratory fitness (Fig. 3g).

When observing physical health data, including average heart rate, exercise load, power to heart rate ratios, we can see increased CRF from mid-June to mid-August, indicating higher loads of training (Fig. 3m). Other confounding factors contribute to apparent changes in cardiovascular health, including changing from highaltitude exercise to sea level (Fig 3j).

We see consistent sleep from March to April (Fig. 3n); this also matches up in the same time frame with increased sustained HRV 
(Fig. 3a), indicating a possible correlation with better sleep and lower stress. There is increased sleep variability from June to July (Fig. 3o), overlapping with the time frame with decreased HRV. We also see a sustained low sleep period relative to baseline (Fig. 3p) and a rebound parasympathetic activity in HRV after the period of sleep deprivation (Fig. 3d).

\subsubsection{User 2: Case Study. The results for User 2 is shown in 4.}

User 2 shows a higher exercise training load period (Fig. 4a) from March to April and a consistent decrease in HRV from March to May. During this period, CRF increases, indicating a possible correlation in HRV decrease that may not be triggered by high mental stress. A significant life event moving from high to low altitude climate shows now decreased CRF over time (Fig. 4d), as what would be expected as the individual has more blood cells in high altitude elevation [26]. The highest HRV variability during the moving event also indicates a potentially stressful period or irregularity in routines. The consistency in exercise load is gradual from June to August (Fig. 4b) after User 2 has settled in the new location, up until the work stressor event in early August. An inflection point (Fig. 4g) shows a sudden decrease in HRV, possibly contributed by work stress.

4.2.5 User 3: Case Study. The results for User 3 is shown in 5.

User 3 data shows a sustained increase in HRV and a sharp increase in sleep hours. While overall sleep is increasing, we see high variability (Fig. 5e) before a major medical exam in early January. In March, a new internal medicine school rotation period began, and sleep became more inconsistent and gradually decreased. In late June, User 3 received a new kitten. We see a sharp increase in HRV (Fig. 5c) and a new consistency in sleep routine (Fig 5g). We hypothesize the new kitten's impact may be decreasing the stress of User 3 and therefore having positive effects on their mental state.

4.2.6 User 4: Case Study. The results for User 4 is shown in 6.

User 4 indicates a life event when a medical school exam is complete. We see a rebound parasympathetic event occur in HRV (Fig. 6a). When user 4 begins a lab rotation and spends much time working over the summer, there is a sustained sleep decrease (Fig 6e). There is also a short-term decrease in HRV (Fig 6b) immediately prior to the start of medical school, indicating there may be stress prior to the school year and finishing lab rotation work. While it looks like User 4 experiences a sudden period of weight loss (Fig $6 c$ ), we recognize that the data accuracy may be an issue when the user is very infrequently taking weight measurements, and the loss may not be an adequate representation of change over time.

\subsection{Discussion}

In this proof of concept study, we propose a platform to assess a user's mental health state quantitatively through analysis of various biological mental health and lifestyle dimensions. We can extract relevant signals from the raw data and convert these into easily interpretable insights to help guide the provider's clinical decisionmaking process. First, the patient's data can be utilized to establish objective baseline measures for various parameters that reflect their current mental health state. We can also objectively collect patient history. Second, the provider and patient can set measurable objectives or SMART goals using these baseline parameters to set lifestyle interventions. Third, progress towards achieving the desired goals can be measured over time, and real-time feedback from the efficacy of any employed interventions (lifestyle modifications, psychotherapy, pharmacologic agents) can be quantitatively assessed. Providers can see sudden shifts as prodromal indications of transition to a "yellow" or "red" zone. Fourth, providers can assess medications and therapy effectiveness based on lifestyle outcomes demonstrated by the data captured. Fifth, providers can make adjustments to any interventions can then be managed to best support the patient's desired outcome.

Providers may use the data obtained through our platform to have a quick overview of their patient's status in various lifestyle biomarkers of their mental condition, as shown in 7. Clinical use by a provider to optimize care for many mental health conditions, such as major depressive disorder (MDD) or ADHD. The current implementation of our platform collects user data reflecting: sleep, physical activity, stress, and focus. Poor sleep and lack of physical activity have been shown to contribute to the development or exacerbation of MDD, so a patient's sleeping patterns and physical activity level would be of interest to the provider caring for a patient with MDD. If this patient had opted to utilize our platform, the provider could view the most clinically relevant life event parameters from our dashboard. In the case of this patient with MDD, the provider could objectively assess the quality and quantity of sleep, the overall amount and intensity of physical activity, and the physiological stress level of the patient. Likewise, a provider treating a patient for ADHD may be more interested in looking at the user's number of phone pickups, total screen time, and productive screen time to reflect their productivity level and distractibility. These data values would establish the patient's baseline, and providers would prioritize which areas they should be focusing on helping the member with the greatest impact on lifestyle and routines. Priorities indicate areas that the user may need the most adjustment to optimize their mental health score.

If the provider prescribed a lifestyle modification for a patient with MDD, such as increasing their amount of physical activity, both the patient's compliance and response to this intervention could be measured through our platform. An increase in step count, stair count, and maximal heart rate from their baseline would reflect a patient's compliance with this intervention, and a concomitant increase in HRV could objectively improve the patient's MDD severity. Similarly, the efficacy of a prescribed pharmacologic agent for this patient's MDD could be objectively assessed by viewing trends in their sleep quantity and quality or HRV. A decrease in the patient's sleep efficiency (total time asleep/total time in bed) and an increase in the number of nighttime awakenings or decrease in HRV may reflect a poor response to the pharmacologic agent, which is valuable data the provider could use to inform their subsequent treatment decisions. The provider may opt to increase the dose of the pharmacologic agent, switch to a different pharmacologic agent, or add additional psychotherapy as some examples. Rather than relying solely on retrospective surveying to assess a patient's mental health state, this type of subjective data can be used in conjunction with the objective data obtained through our platform to enhance the resolution of which a patient's mental health state can be accurately defined. Ultimately, we envision providers using our EMA-based platform to augment their clinical decision-making 


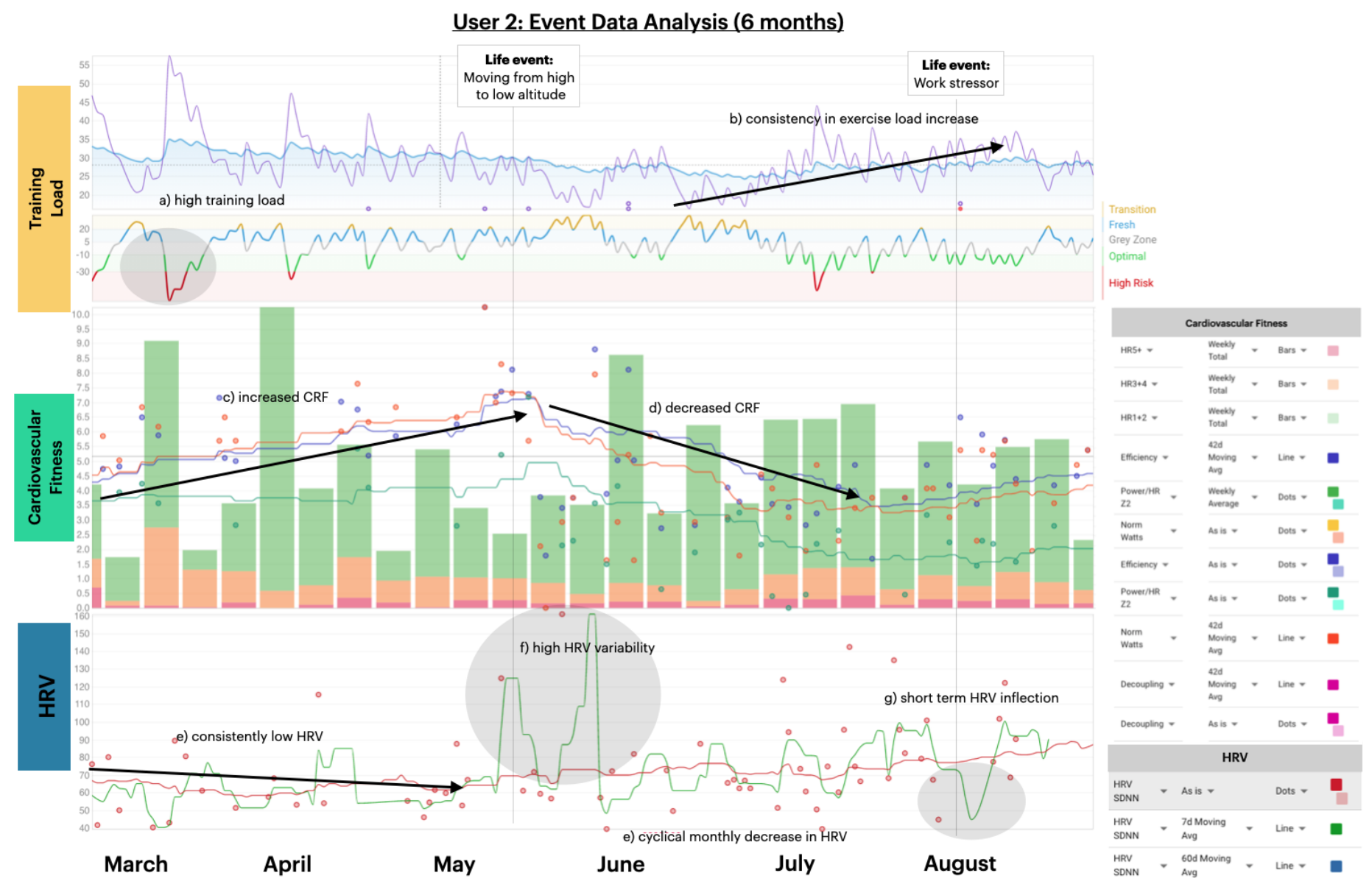

Figure 4: User 2 Data Trends: We analyzed six months of data for User 1, capturing trends in HRV, exercise load, and CRF. Labels a through g provide relevant trends and insights from both 7-day moving averages and 42-day moving averages.

with the support of empiric evidence to optimize the care for mental health patients and improve patient outcomes.

\section{CONCLUSIONS AND FUTURE OPPORTUNITIES}

Continuous sensing methods provide extensive insight about an individual's day-to-day functioning and are easily accessible technology, as many users already have sensor technologies such as smart-phones or wearable devices. However, it is essential to note that the quality of the data captured may be variable. No sensing system is entirely accurate, but the large amounts of temporal data provide a set of surrogate biomarkers to estimate a user's mental health state, screen for disorders, provide treatment knowledge, and prescribe lifestyle changes.

Many opportunities remain for identifying patterns in data to make more accurate diagnoses including understanding a user's lifestyle data related to mental health. We see opportunities for further investigation of the mental health estimation framework related to lifestyle events in a clinical setting. Providers and applications can increase their effectiveness by utilizing the lifestyle activity to analyze factors that may be contributing to changes in mental health. Ultimately, this will help the user and the provider make better recommendations for improving the user's situation.

We must also note that using a data-driven approach to mental health measurements will also bring several considerations around data privacy, ownership, and confidentiality that must be investigated in depth. While there are barriers regarding privacy of constant tracking, many patients are motivated to understand their mental health state and see measurable progress.

Lastly, it is important to note that estimation research in itself does not produce actionable next steps. Estimation creates the basis by which we can measure states and observe patterns, but ultimately there must be guidance for both users and providers to know recommended next steps in the user's care journey. The concept of guidance in mental health tracking brings forward the need for personal mental health navigation as an area for further investigation $[55,66]$. 


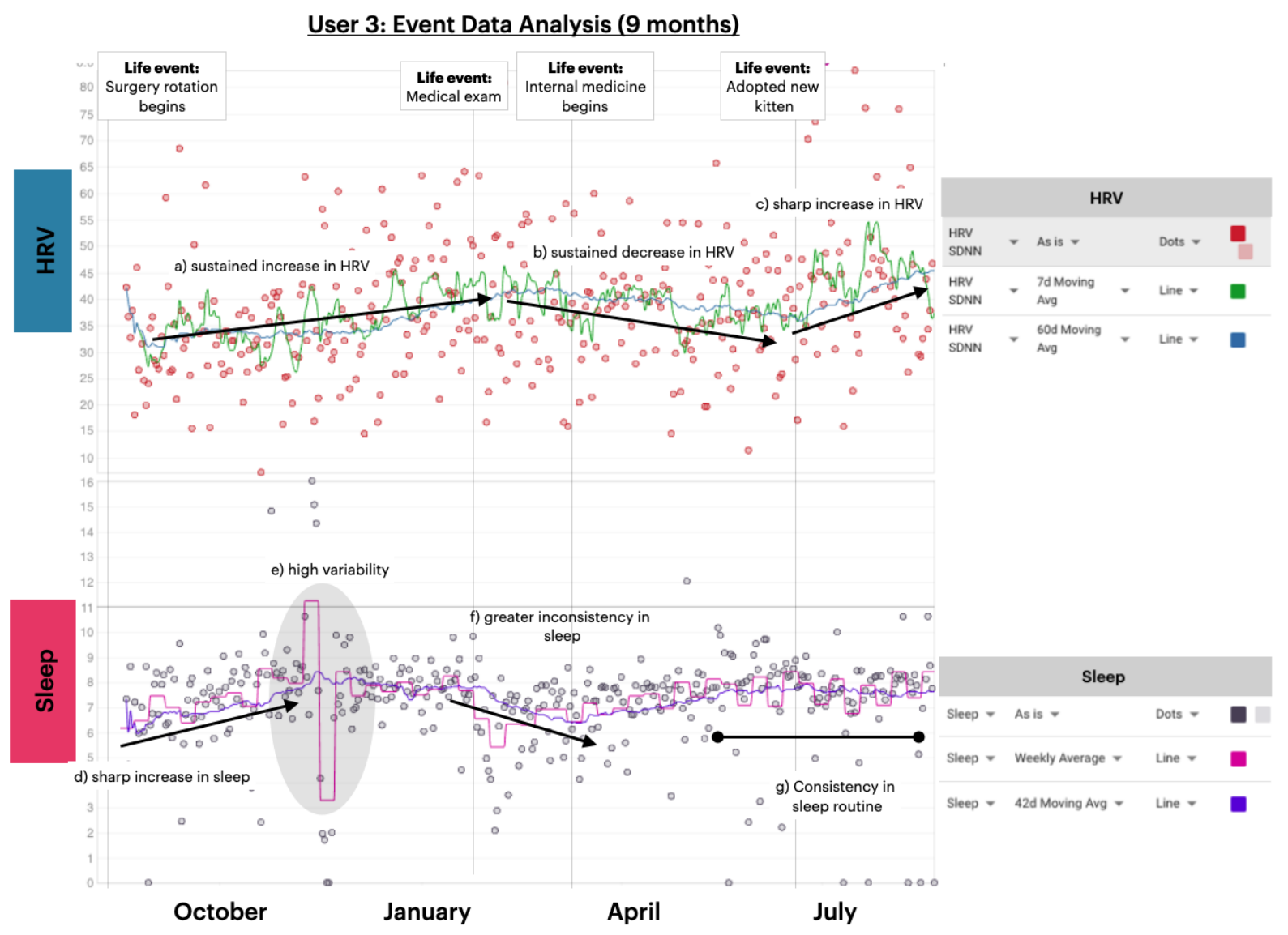

Figure 5: User 3 Data Trends: We analyzed nine months of data for User 3, capturing trends in HRV and sleep. Labels a through g provide relevant trends and insights from both 7-day moving averages and 42-day moving averages.

\section{REFERENCES}

[1] [n.d.]. 21\% of Americans use a smart watch or fitness tracker $\mid$ Pew Research Center. https://www.pewresearch.org/fact-tank/2020/01/09/about-one-in-fiveamericans-use-a-smart-watch-or-fitness-tracker/

[2] [n.d.]. Apple, Microsoft and Google to test new standard for patient access to digital health data | TechCrunch. https://techcrunch.com/2019/07/30/applemicrosoft-and-google-to-test-new-standard-for-patient-access-to-digitalhealth-data/

[3] [n.d.]. DSM-5 ${ }^{\circledR}$ Made Easy: The Clinician's Guide to Diagnosis - James Morrison - Google Books. https://books.google.com/books?hl=en\&lr= \&id=Vn38AgAAQBAJ\&oi=fnd\&pg=PP1\&dq=DSM+5+simplified+book\&ots= AsrcMQmtqv\&sig=rpOQkdCe7usNbBThSmQ5rRwDppQ\#v=onepage\&q\&f= false

[4] [n.d.]. Reinforcement Learning, second edition: An Introduction - Richard S. Sutton, Andrew G. Barto - Google Books. https://books.google.com/books?hl=en\& $\mathrm{l} r=\& \mathrm{id}=\mathrm{uWV0DwAAQBAJ} \& \mathrm{oi}=$ fnd $\& \mathrm{pg}=\mathrm{PR} 7 \& \mathrm{dq}=$ reinforcement+learning \& ots=mipNt520n1\&sig=Ruxs3HQf9WelRBvtgf3RVc6UZSA\#v=onepage \&q= reinforcement $\% 20$ learning $\& \mathrm{f}=$ false

[5] [n.d.]. RescueTime - Your Daily dashboard. https://www.rescuetime.com/ dashboard

[6] [n.d.]. Smart lighting | Philips Hue. https://www.philips-hue.com/en-us

[7] [n.d.]. Track daily activity with Apple Watch - Apple Support. https://support.apple.com/guide/watch/track-daily-activity-with-applewatch-apd3bf6d85a6/watchos

[8] [n.d.]. Welcome to the HL7 FHIR Foundation. https://fhir.org/

[9] [n.d.]. • US smartphone ownership 2021 | Statista. https://www.statista.com/ statistics/219865/percentage-of-us-adults-who-own-a-smartphone/

[10] Anna Z. Antosik-Wójcińska, Monika Dominiak, Magdalena Chojnacka, Katarzyna Kaczmarek-Majer, Karol R. Opara, Weronika Radziszewska, Anna
Olwert, and Łukasz Święcicki. 2020. Smartphone as a monitoring tool for bipolar disorder: a systematic review including data analysis, machine learning algorithms and predictive modelling. International fournal of Medical Informatics 138 (6 2020), 104131. https://doi.org/10.1016/j.ijmedinf.2020.104131

[11] Chiara Baglioni, Gemma Battagliese, Bernd Feige, Kai Spiegelhalder, Christoph Nissen, Ulrich Voderholzer, Caterina Lombardo, and Dieter Riemann. 2011. Insomnia as a predictor of depression: A meta-analytic evaluation of longitudinal epidemiological studies. Fournal of Affective Disorders 135, 1-3 (12 2011), 10-19. https://doi.org/10.1016/J.JAD.2011.01.011

[12] Theodore P. Beauchaine, Lisa Gatzke-Kopp, Emily Neuhaus, Jane Chipman, M. Jamila Reid, and Carolyn Webster-Stratton. 2013. Sympathetic- and parasympathetic-linked cardiac function and prediction of externalizing behavior, emotion regulation, and prosocial behavior among preschoolers treated for ADHD. Journal of consulting and clinical psychology 81, 3 (2013), 481-493. https://doi.org/10.1037/A0032302

[13] Erin E. Brannon, Christopher C. Cushing, Christopher J. Crick, and Tarrah B. Mitchell. 2016. The promise of wearable sensors and ecological momentary assessment measures for dynamical systems modeling in adolescents: a feasibility and acceptability study. Translational Behavioral Medicine 6, 4 (12 2016), 558. https://doi.org/10.1007/S13142-016-0442-4

[14] Reiner Buchhorn, Annette Conzelmann, Christian Willaschek, Dagmar Störk, Regina Taurines, and Tobias J. Renner. 2012. Heart rate variability and methylphenidate in children with ADHD. ADHD Attention Deficit and Hyperactivity Disorders 2012 4:2 4, 2 (2 2012), 85-91. https://doi.org/10.1007/S12402012-0072-8

[15] Terry C. Camacho, Robert E. Roberts, Nancy B. Lazarus, George A. Kaplan, and Richard D. Cohen. 1991. Physical Activity and Depression: Evidence from the Alameda County Study. American fournal of Epidemiology 134, 2 (7 1991), 220-231. https://doi.org/10.1093/OXFORDJOURNALS.AJE.A116074 


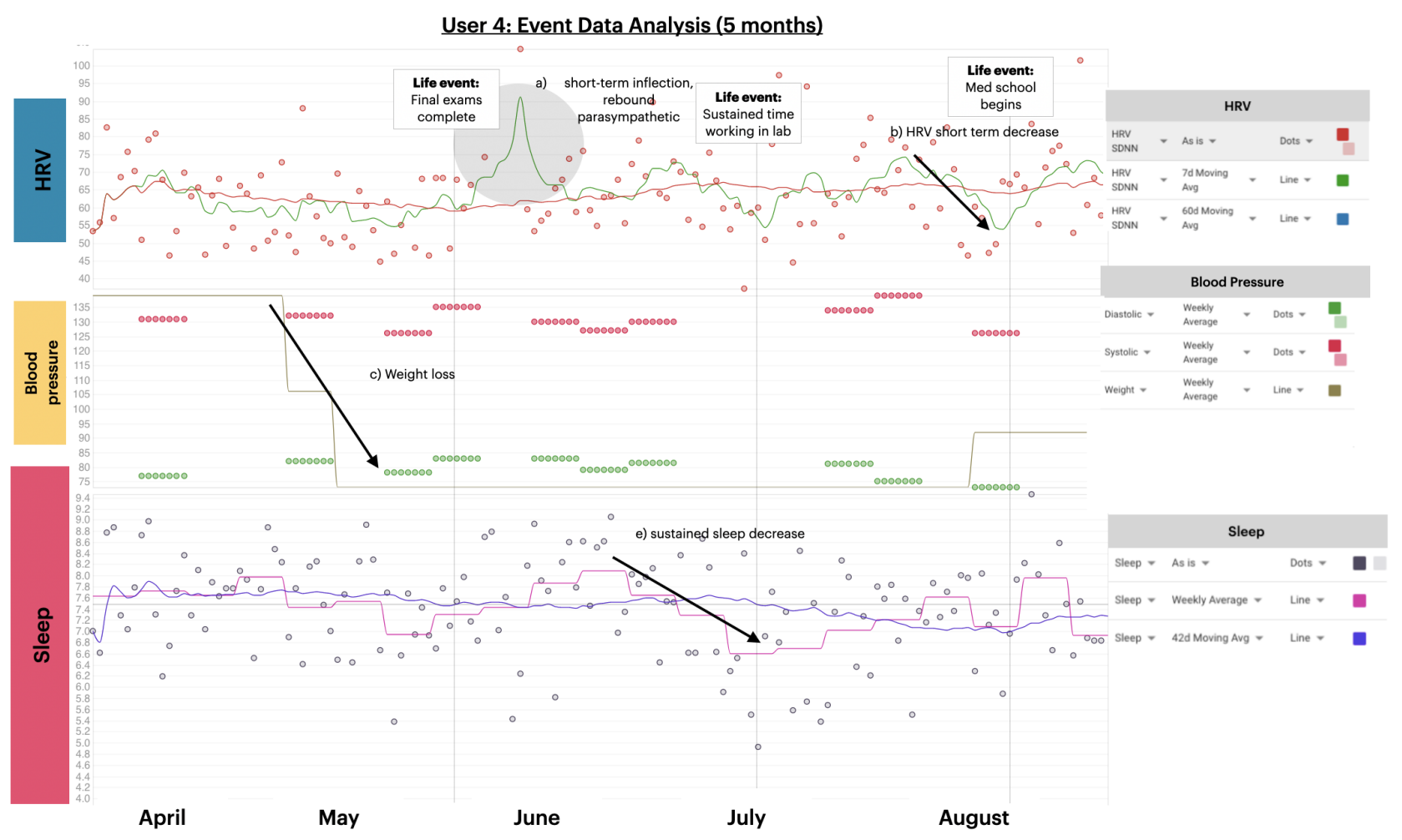

Figure 6: User 4 Data Trends: We analyzed five months of data for User 4, capturing trends in HRV, blood pressure, weight, CRF, and sleep. Labels a through d provide relevant trends and insights from both 7-day moving averages and 42-day moving averages.

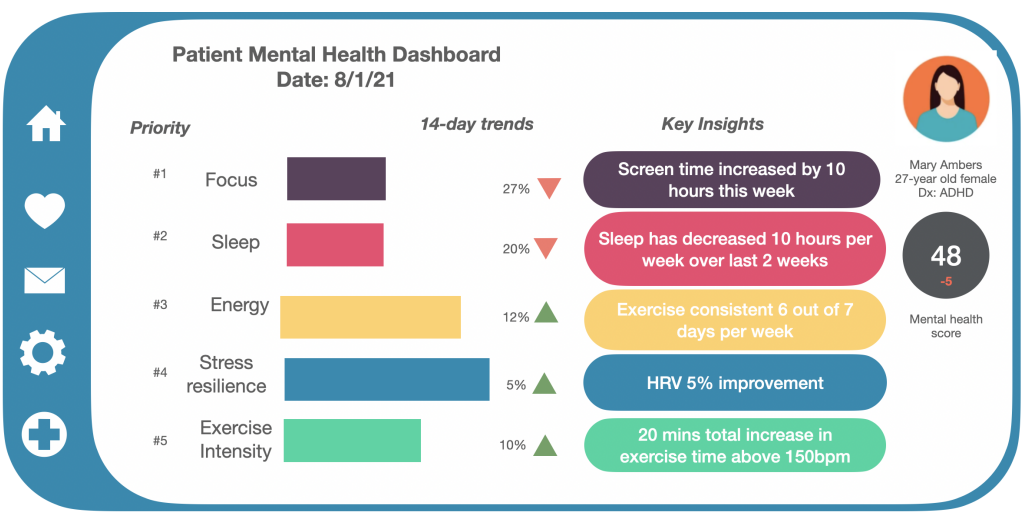

Figure 7: Provider Dashboard: This dashboard shows outputs of how a provider could observe key trends as it relates to lifestyle impact to mental health on an ongoing basis.

[16] Hui Cao, Qingwen Qian, Tingting Weng, Changjiang Yuan, Ying Sun, Hui Wang, and Fangbiao Tao. 2011. Screen time, physical activity and mental health among urban adolescents in China. Preventive Medicine 53, 4-5 (10 2011), 316-320. https://doi.org/10.1016/J.YPMED.2011.09.002

[17] John A. Chalmers, Daniel S. Quintana, Maree J.-Anne Abbott, and Andrew H. Kemp. 2014. Anxiety Disorders are Associated with Reduced Heart Rate Variability: A Meta-Analysis. Frontiers in Psychiatry 5, JUL (2014). https: //doi.org/10.3389/FPSYT.2014.00080
[18] John S. Y. Chan, Guanmin Liu, Danxia Liang, Kanfeng Deng, Jiamin Wu, and Jin H. Yan. 2018. Special Issue - Therapeutic Benefits of Physical Activity for Mood: A Systematic Review on the Effects of Exercise Intensity, Duration, and Modality. https://doi.org/10.1080/00223980.2018.1470487 153, 1 (1 2018), 102-125. https://doi.org/10.1080/00223980.2018.1470487

[19] Sammi R. Chekroud, Ralitza Gueorguieva, Amanda B. Zheutlin, Martin Paulus, Harlan M. Krumholz, John H. Krystal, and Adam M. Chekroud. 2018. Association between physical exercise and mental health in 1.2 million individuals in the 
USA between 2011 and 2015: a cross-sectional study. The Lancet Psychiatry 5, 9 (9 2018), 739-746. https://doi.org/10.1016/S2215-0366(18)30227-X

[20] Zhenyu Chen. 2013. Unobtrusive sleep monitoring using smartphones. IEEE Xplore (2013).

[21] Emma Childs and Harriet de Wit. 2014. Regular exercise is associated with emotional resilience to acute stress in healthy adults. Frontiers in Physiology 5 (2014). https://doi.org/10.3389/FPHYS.2014.00161

[22] Karmel W. Choi, Chia-Yen Chen, Murray B. Stein, Yann C. Klimentidis, Min-Jung Wang, Karestan C. Koenen, Jordan W. Smoller, and for the Major Depressive Disorder Working Group of the Psychiatric Genomics Consortium. 2019. Assessment of Bidirectional Relationships Between Physical Activity and Depression Among Adults: A 2-Sample Mendelian Randomization Study. FAMA Psychiatry 76, 4 (4 2019), 399-408. https://doi.org/10.1001/JAMAPSYCHIATRY.2018.4175

[23] Charles K Chui and Guanrong Chen. [n.d.]. Kalman Filtering. ([n. d.]).

[24] Annika Clamor, Tania M. Lincoln, Julian F. Thayer, and Julian Koenig. 2016. Resting vagal activity in schizophrenia: Meta-analysis of heart rate variability as a potential endophenotype. The British fournal of Psychiatry 208, 1 (1 2016) 9-16. https://doi.org/10.1192/BJP.BP.114.160762

[25] Rachel C Colley, Tracey Bushnik, and Kellie Langlois. [n.d.]. Health Reports. ([n. d.]). https://doi.org/10.25318/82-003-x202000600001-eng

[26] Angelo D’Alessandro, Travis Nemkov, Kaiqi Sun, Hong Liu, Anren Song, Andrew A. Monte, Andrew W. Subudhi, Andrew T. Lovering, Daniel Dvorkin, Colleen G. Julian, Christopher G. Kevil, Gopi K. Kolluru, Sruti Shiva, Mark T. Gladwin, Yang Xia, Kirk C. Hansen, and Robert C. Roach. 2016. AltitudeOmics: Red Blood Cell Metabolic Adaptation to High Altitude Hypoxia. Fournal of Proteome Research 15, 10 (10 2016), 3883-3895. https://doi.org/10.1021/ACS.JPROTEOME. 6B00733

[27] Kenneth R Fox. 1999. The influence of physical activity on mental wellbeing. Public Health Nutrition 2, 3a (1999), 411-418. https://doi.org/10.1017/ S1368980099000567

[28] Daniel Freeman, Bryony Sheaves, Guy M. Goodwin, Ly Mee Yu, Alecia Nickless, Paul J. Harrison, Richard Emsley, Annemarie I. Luik, Russell G. Foster, Vanashree Wadekar, Christopher Hinds, Andrew Gumley, Ray Jones, Stafford Lightman, Steve Jones, Richard Bentall, Peter Kinderman, Georgina Rowse, Traolach Brugha, Mark Blagrove, Alice M. Gregory, Leanne Fleming, Elaine Walklet, Cris Glazebrook, E. Bethan Davies, Chris Hollis, Gillian Haddock, Bev John, Mark Coulson, David Fowler, Katherine Pugh, John Cape, Peter Moseley, Gary Brown, Claire Hughes, Marc Obonsawin, Sian Coker, Edward Watkins, Matthias Schwannauer, Kenneth MacMahon, A. Niroshan Siriwardena, and Colin A. Espie. 2017. The effects of improving sleep on mental health (OASIS): a randomised controlled trial with mediation analysis. The Lancet Psychiatry 4, 10 (10 2017), 749-758. https://doi.org/10.1016/S2215-0366(17)30328-0

[29] Silvana Galderisi, Andreas Heinz, Marianne Kastrup, Julian Beezhold, and Norman Sartorius. 2015. Toward a new definition of mental health. World Psychiatry 14, 2 (6 2015), 231. https://doi.org/10.1002/WPS.20231

[30] Stephen Gillinov, Muhammad Etiwy, Robert Wang, Gordon Blackburn, Dermot Phelan, A. Marc Gillinov, Penny Houghtaling, Hoda Javadikasgari, and Milind Y. Desai. 2017. Variable accuracy of wearable heart rate monitors during aerobic exercise. Medicine and Science in Sports and Exercise 49, 8 (8 2017), 1697-1703. https://doi.org/10.1249/MSS.0000000000001284

[31] Google. [n.d.]. Google Nest. https://store.google.com/us/category/connected home?

[32] Sydney Heiss, Bronya Vaschillo, Evgeny G. Vaschillo, C. Alix Timko, and Julia M. Hormes. 2021. Heart rate variability as a biobehavioral marker of diverse psychopathologies: A review and argument for an "ideal range". Neuroscience \& Biobehavioral Reviews 121 (2 2021), 144-155. https://doi.org/10.1016/J.NEUBIOREV. 2020.12.004

[33] Aaron S. Heller, Tracey C. Shi, C. E. Chiemeka Ezie, Travis R. Reneau, Lara M. Baez, Conor J. Gibbons, and Catherine A. Hartley. 2020. Association between realworld experiential diversity and positive affect relates to hippocampal-striatal functional connectivity. Nature Neuroscience 23, 7 (7 2020), 800-804. https: //doi.org/10.1038/s41593-020-0636-4

[34] David Hernando, Surya Roca, Jorge Sancho, Álvaro Alesanco, and Raquel Bailón 2018. Validation of the Apple Watch for Heart Rate Variability Measurements during Relax and Mental Stress in Healthy Subjects. Sensors (Basel, Switzerland) 18, 8 (8 2018). https://doi.org/10.3390/S18082619

[35] Kit Huckvale, Svetha Venkatesh, and Helen Christensen. 2019. Toward clinical digital phenotyping: a timely opportunity to consider purpose, quality, and safety. npj Digital Medicine 2, 1 (12 2019), 88. https://doi.org/10.1038/s41746-019-0166-1

[36] Thomas R. Insel. 2017. Digital Phenotyping. JAMA 318, 13 (10 2017), 1215 https://doi.org/10.1001/jama.2017.11295

[37] Ramesh Jain and Laleh Jalali. 2014. Objective self. IEEE Multimedia 21, 4 (2014), 100-110. https://doi.org/10.1109/MMUL.2014.63

[38] Laleh Jal, Hyungik Oh, Siripen Pongpaichet, Ramesh Jain, Laleh Jalali, Da Huo, and Mengfan Tang. 2014. Personicle: Personal Chronicle of Life Events electrical characterization of devices View project CueNet: Progressive Discovery of Context View project Personicle: Personal Chronicle of Life Events. (2014). https://www.researchgate.net/publication/268523858

[39] Karine Alexandra Del Rio João, Saul Neves de Jesus, Cláudia Carmo, and Patríci Pinto. 2018. The impact of sleep quality on the mental health of a non-clinical population. Sleep Medicine 46 (6 2018), 69-73. https://doi.org/10.1016/J.SLEEP. 2018.02.010

[40] Aaron Kandola, Garcia Ashdown-Franks, Joshua Hendrikse, Catherine M. Sabiston, and Brendon Stubbs. 2019. Physical activity and depression: Towards understanding the antidepressant mechanisms of physical activity. Neuroscience \& Biobehavioral Reviews 107 (12 2019), 525-539. https://doi.org/10.1016/ J.NEUBIOREV.2019.09.040

[41] Andrew H. Kemp, Daniel S. Quintana, Marcus A. Gray, Kim L. Felmingham, Kerri Brown, and Justine M. Gatt. 2010. Impact of Depression and Antidepressant Treatment on Heart Rate Variability: A Review and Meta-Analysis. Biological Psychiatry 67, 11 (6 2010), 1067-1074. https://doi.org/10.1016/J.BIOPSYCH.2009. 12.012

[42] Julian Koenig, Andrew H. Kemp, Nicole R. Feeling, Julian F. Thayer, and Michael Kaess. 2016. Resting state vagal tone in borderline personality disorder: A metaanalysis. Progress in Neuro-Psychopharmacology and Biological Psychiatry 64 (1 2016), 18-26. https://doi.org/10.1016/J.PNPBP.2015.07.002

[43] Kurt Kroenke, Robert L. Spitzer, and Janet B. W. Williams. 2001. The PHQ-9. fournal of General Internal Medicine 16, 9 (9 2001), 606-613. https://doi.org/10. 1046/J.1525-1497.2001.016009606.X

[44] Daniel M. LeBouthillier and Gordon J.G. Asmundson. 2017. The efficacy of aerobic exercise and resistance training as transdiagnostic interventions for anxietyrelated disorders and constructs: A randomized controlled trial. Fournal of Anxiety Disorders 52 (12 2017), 43-52. https://doi.org/10.1016/J.JANXDIS.2017.09.005

[45] Gadi Lissak. 2018. Adverse physiological and psychological effects of screen time on children and adolescents: Literature review and case study. Environmental Research 164 (7 2018), 149-157. https://doi.org/10.1016/J.ENVRES.2018.01.015

[46] Sumit Majumder and M. Jamal Deen. 2019. Smartphone sensors for health monitoring and diagnosis. https://doi.org/10.3390/s19092164

[47] Sumit Majumder, Tapas Mondal, and M. Jamal Deen. 2017. Wearable Sensors for Remote Health Monitoring. Sensors 2017, Vol. 17, Page 130 17, 1 (1 2017), 130. https://doi.org/10.3390/S17010130

[48] K A Matthews, K L Woodall, and M T Allen. 1993. Cardiovascular reactivity to stress predicts future blood pressure status. Hypertension 22, 4 (1993), 479-485. https://doi.org/10.1161/01.HYP.22.4.479

[49] Nazar Mazurak, Paul Enck, Eric Muth, Martin Teufel, and Stephan Zipfel. 2011. Heart rate variability as a measure of cardiac autonomic function in anorexia nervosa: A review of the literature. European Eating Disorders Review 19, 2 (3 2011), 87-99. https://doi.org/10.1002/ERV.1081

[50] Richard Miller. 2020. How Much Does A Psychiatrist Cost? . https://www. betterhelp.com/advice/psychiatry/how-much-does-a-psychiatrist-cost/

[51] Breteler MJ, Janssen JH, Spiering W, Kalkman CJ, van Solinge WW, and Dohmen DA. 2019. Measuring Free-Living Physical Activity With Three Commercially Available Activity Monitors for Telemonitoring Purposes: Validation Study. $7 M I R$ formative research 3, 2 (2019). https://doi.org/10.2196/11489

[52] David C. Mohr, Mi Zhang, and Stephen M. Schueller. 2017. Personal Sensing: Understanding Mental Health Using Ubiquitous Sensors and Machine Learning. https://doi.org/10.1146/annurev-clinpsy-032816-044949 13 (5 2017), 23-47. https: //doi.org/10.1146/ANNUREV-CLINPSY-032816-044949

[53] Nidal Moukaddam and Ashutosh Sabharwal. 2021. Data-Driven: How Technology Can Improve Psychiatric Practice. Psychiatric Times (2021), 45. https://www.psychiatrictimes.com/view/data-driven-how-technology-canimprove-psychiatric-practice

[54] Klaus Munkholm, Maj Vinberg, and Lars Vedel Kessing. 2013. Cytokines in bipolar disorder: A systematic review and meta-analysis. Fournal of Affective Disorders 144, 1-2 (1 2013), 16-27. https://doi.org/10.1016/J.JAD.2012.06.010

[55] Nitish Nag and Ramesh Jain. 2019. A Navigational Approach to Health: Actionable Guidance for Improved Quality of Life. Computer 52, 4 (4 2019), 12-20. https: //doi.org/10.1109/MC.2018.2883280

[56] Nitish Nag, Vaibhav Pandey, Preston J. Putzel, Hari Bhimaraju, Srikanth Krishnan, and Ramesh Jain. 2018. Cross-modal health state estimation. In MM 2018 Proceedings of the 2018 ACM Multimedia Conference. Association for Computing Machinery, Inc, New York, New York, USA, 1993-2002. https://doi.org/10.1145/ 3240508.3241913

[57] Aravind Natarajan, Alexandros Pantelopoulos, Hulya Emir-Farinas, and Pradeep Natarajan. 2020. Heart rate variability with photoplethysmography in 8 million individuals: a cross-sectional study. The Lancet Digital Health 2, 12 (12 2020), e650-e657. https://doi.org/10.1016/S2589-7500(20)30246-6

[58] National Institute of Mental Health. 2021. Research Domain Criteria Initiative. National Institute of Mental Health (2021). xhttps://www.nimh.nih.gov/research/ research-funded-by-nimh/rdoc

[59] Hyungik Oh and Ramesh Jain. 2017. From Multimedia Logs to Personal Chronicles. Proceedings of the 25th ACM international conference on Multimedia (2017). https://doi.org/10.1145/3123266

[60] Scott A. Paluska and Thomas L. Schwenk. 2012. Physical Activity and Mental Health. Sports Medicine 2000 29:3 29, 3 (9 2012), 167-180. https://doi.org/10.2165 
00007256-200029030-00003

[61] Emily M. Paolucci, Dessi Loukov, Dawn M.E. Bowdish, and Jennifer J. Heisz. 2018 Exercise reduces depression and inflammation but intensity matters. Biological Psychology 133 (3 2018), 79-84. https://doi.org/10.1016/J.BIOPSYCHO.2018.01.015

[62] Eunil Park, Ki Joon Kim, and Sang Jib Kwon. 2016. Understanding the emergence of wearable devices as next-generation tools for health communication. Information Technology \&amp; People 29, 4 (2016), 717-732. https://doi.org/10.1108/ITP04-2015-0096

[63] Tiina Paunio, Tellervo Korhonen, Christer Hublin, Markku Partinen, Karoliina Koskenvuo, Markku Koskenvuo, and Jaakko Kaprio. 2015. Poor sleep predicts symptoms of depression and disability retirement due to depression. Fournal of Affective Disorders 172 (2 2015), 381-389. https://doi.org/10.1016/J.JAD.2014.10. 002

[64] Alejandro Porras-Segovia, Rosa María Molina-Madueño, Sofian Berrouiguet, Jorge López-Castroman, Maria Luisa Barrigón, María Sandra Pérez-Rodríguez, José Heliodoro Marco, Isaac Díaz-Oliván, Santiago de León, Philippe Courtet, Antonio Artés-Rodríguez, and Enrique Baca-García. 2020. Smartphone-based ecological momentary assessment (EMA) in psychiatric patients and student controls: A real-world feasibility study. Fournal of Affective Disorders 274 (9 2020), 733-741. https://doi.org/10.1016/J.JAD.2020.05.067

[65] Honghui Qi and John B. Moore. 2002. Direct Kalman filtering approach for GPS/INS integration. IEEE Trans. Aerospace Electron. Systems 38, 2 (4 2002), 687-693. https://doi.org/10.1109/TAES.2002.1008998

[66] Amir M. Rahmani, Jocelyn Lai, Salar Jafarlou, Asal Yunusova, Alex. P. Rivera, Sina Labbaf, Sirui Hu, Arman Anzanpour, Nikil Dutt, Ramesh Jain, and Jessica L. Borelli. 2020. Personal Mental Health Navigator: Harnessing the Power of Data Personal Models, and Health Cybernetics to Promote Psychological Well-being. (12 2020). https://arxiv.org/abs/2012.09131v1

[67] Andrew G Reece and Christopher M Danforth. 2017. Instagram photos reveal predictive markers of depression. EPf Data Science 2017 6:1 6, 1 (8 2017), 1-12. https://doi.org/10.1140/EPJDS/S13688-017-0110-Z

[68] Dieter Riemann and Ulrich Voderholzer. 2003. Primary insomnia: a risk factor to develop depression? Fournal of Affective Disorders 76, 1-3 (9 2003), 255-259. https://doi.org/10.1016/S0165-0327(02)00072-1

[69] Daniel M Roberts, Margeaux M Schade, Gina M Mathew, Daniel Gartenberg, and Orfeu M Buxton. 2020. Detecting sleep using heart rate and motion data from multisensor consumer-grade wearables, relative to wrist actigraphy and polysomnography. Sleep 43, 7 (2020), 1-19. https://doi.org/10.1093/SLEEP/ ZSAA045

[70] Amita Sehgal and Emmanuel Mignot. 2011. Genetics of Sleep and Sleep Disorders. Cell 146, 2 (7 2011), 194-207. https://doi.org/10.1016/J.CELL.2011.07.004

[71] Saul Shiffman, Arthur A. Stone, and Michael R. Hufford. 2008. Ecological Momentary Assessment. http://dx.doi.org/10.1146/annurev.clinpsy.3.022806.091415 (3 2008), 1-32. https://doi.org/10.1146/ANNUREV.CLINPSY.3.022806.091415

[72] Jinyoung Shin, Jin Young Park, and Jaekyung Choi. 2018. Long-term exposure to ambient air pollutants and mental health status: A nationwide population-based cross-sectional study. PLOS ONE 13, 4 (4 2018), e0195607. https://doi.org/10. 1371/JOURNAL.PONE.0195607

[73] Lucas Raphael Bento Silva, Camila Simões Seguro, Camila Grasiele Araújo de Oliveira, Paulo Otávio Silva Santos, Jordana Campos Martins de Oliveira, Luiz Fernando Martins de Souza Filho, Célio Antônio de Paula Júnior, Paulo Gentil, and Ana Cristina Silva Rebelo. 2020. Physical Inactivity Is Associated With Increased Levels of Anxiety, Depression, and Stress in Brazilians During the COVID-19 Pandemic: A Cross-Sectional Study. Frontiers in Psychiatry 0 (11 2020), 1257. https://doi.org/10.3389/FPSYT.2020.565291

[74] Stanford Medicine Snyder Lab. 2021. Personal Molecular and Medical Phenotypes. Stanford Medicine (2021). https://med.stanford.edu/snyderlab/ipop.html

[75] PHYLLIS K. STEIN, PETER P. DOMITROVICH, NELSON HUI, PENTTI RAUTAHARJU, and JOHN GOTTDIENER. 2005. Sometimes Higher Heart Rate Variability Is Not Better Heart Rate Variability: Results of Graphical and Nonlinear Analyses. Fournal of Cardiovascular Electrophysiology 16, 9 (9 2005), 954-959. https://doi.org/10.1111/J.1540-8167.2005.40788.X

[76] William J. Strawbridge, Stéphane Deleger, Robert E. Roberts, and George A Kaplan. 2002. Physical Activity Reduces the Risk of Subsequent Depression for Older Adults. American fournal of Epidemiology 156, 4 (8 2002), 328-334. https://doi.org/10.1093/AJE/KWF047

[77] John Torous and Adam C. Powell. 2015. Current research and trends in the use of smartphone applications for mood disorders. Internet Interventions 2, 2 (5 2015), 169-173. https://doi.org/10.1016/j.invent.2015.03.002

[78] Conny M. A. van Ravenswaaij-Arts. 1993. Heart Rate Variability. Annals of Internal Medicine 118, 6 (3 1993), 436. https://doi.org/10.7326/0003-4819-118-6199303150-00008

[79] William H. Walker, James C. Walton, A. Courtney DeVries, and Randy J. Nelson 2020. Circadian rhythm disruption and mental health. Translational Psychiatry 2020 10:1 10, 1 (1 2020), 1-13. https://doi.org/10.1038/s41398-020-0694-0

[80] Le Meur Y, Pichon A, Schaal K, Schmitt L, Louis J, Gueneron J, Vidal PP, and Hausswirth C. 2013. Evidence of parasympathetic hyperactivity in functionally overreached athletes. Medicine and science in sports and exercise 45, 11 (2013),
2061-2071. https://doi.org/10.1249/MSS.0B013E3182980125 